\title{
ON THE NUMBER OF PARTITIONS OF A NUMBER INTO UNEQUAL PARTS $\left({ }^{\mathrm{i}}\right)$
}

\author{
BY \\ LOO-KENG HUA
}

1. Introduction. Let $q(n)$ be the number of partitions of an integer $n$ into unequal parts, or into odd parts $\left({ }^{2}\right)$. Then

$$
\begin{aligned}
f(x) & =1+\sum_{n=1}^{\infty} q(n) x^{n}=(1+x)\left(1+x^{2}\right)\left(1+x^{3}\right) \cdots \\
& =\frac{1}{(1-x)\left(1-x^{3}\right)\left(1-x^{5}\right) \cdots} .
\end{aligned}
$$

Hardy and Ramanujan $\left({ }^{3}\right)$ indicated that by their fundamental analytic method one can obtain the following result:

$$
\begin{aligned}
q(n)= & \frac{1}{2^{1 / 2}} \frac{d}{d n} J_{0}\left[i \pi\left\{\frac{1}{3}\left(n+\frac{1}{24}\right)\right\}^{1 / 2}\right] \\
& +2^{1 / 2} \cos \left(\frac{2}{3} \pi n-\frac{1}{9} \pi\right) \frac{d}{d n} J_{2}\left[\frac{1}{3} i \pi\left\{\frac{1}{3}\left(n+\frac{1}{24}\right)\right\}^{1 / 2}\right]+\cdots \\
& + \text { to }\left[\alpha n^{1 / 2}\right] \text { terms }+O(1)
\end{aligned}
$$

where $\alpha$ is an arbitrary constant. This result is less satisfactory than that concerning the number $p(n)$ of partitions (unrestricted) of $n$, since in the latter case the error term approaches zero with increasing $n$. Recently Rademacher $\left({ }^{4}\right)$ obtained an equality for $p(n)$. The object of the present paper is to find an equality for $q(n)$. The work of this paper is a straightforward application of Hardy-Ramanujan's method with two modifications. These modifications are Kloosterman's sum and Rademacher's "Farey dissection of infinite order."

The present method may also be applied to find the explicit formula for

$$
\sum_{x=1}^{[n / 2]} p\left(n-x^{2}\right)
$$

where $p(n)$ is the number of unrestricted partitions of $n$.

Presented to the Society, April 27, 1940; received by the editors January 9, 1941.

(1) This paper was accepted by Acta Arithmetica before the war.

(2) Cf. MacMahon, Combinatory Analysis, vol. 2, 1916, p. 11.

(3) Proceedings of the London Mathematical Society, (2), vol. 17 (1918), pp. 75-115.

(4) Proceedings of the London Mathematical Society, (2), vol. 43 (1937), pp. 241-254. 
2. Statement of the result. Let

$$
\epsilon_{h, k}=\left\{\begin{array}{cc}
\exp \left(-\pi i\left(\frac{\left(h^{\prime 2}-1\right)}{8}\left(\frac{1-h h^{\prime}}{k}-1\right)+\frac{h^{\prime}\left(1-h h^{\prime}\right)}{8 k}\right.\right. & \\
\left.\left.+\frac{1}{24}\left(k+\frac{1-h h^{\prime}}{k}\right)\left(h h^{\prime 2}-h^{\prime}-h\right)\right)\right), & \text { for } 2 \mid k, \\
\exp \left(\frac{\pi i}{24}\left(k+\frac{1-h h^{\prime}}{k}\right)\left(h+h^{\prime}-h^{2} h^{\prime}\right)\right), & \text { for } 2 \nmid k, 2 \nmid h, \\
\exp \left(-\frac{\pi i}{8}\left(k^{2}-1-h k+\frac{1}{3}\left(h+h^{\prime}\right)\left(h h^{\prime} k-\frac{h h^{\prime}-1}{k}\right)\right)\right), & \text { for } 2 \nmid k, 2 \mid h,
\end{array}\right.
$$

and

$$
\omega_{h, k}= \begin{cases}\epsilon_{h, k} \exp \left(-\frac{\pi i}{12 k}\left(h+h^{\prime}\right)\right), & \text { for } 2 \mid k, \\ \epsilon_{h, k} \exp \left(-\frac{\pi i}{24 k}\left(2 h-h^{\prime}\right)\right), & \text { for } 2 \nmid k,\end{cases}
$$

where $h h^{\prime} \equiv 1(\bmod k), h \equiv h^{\prime}(\bmod 2)$.

ThEOREM. The number of partitions of an integer $n$ into unequal parts is given by

$$
q(n)=\frac{1}{2^{1 / 2}} \sum_{k=1, k \text { udd }}^{\infty} \sum_{(h, k)=1,0<h \leqq k} \omega_{h, k} e^{-2 \pi i h n / k} \frac{d}{d n} J_{0}\left(\frac{i \pi}{k}\left\{\frac{2}{3}\left(n+\frac{1}{24}\right)\right\}^{1 / 2}\right),
$$

where $J_{0}(x)$ is the Bessel function of the 0 th order.

3. Farey dissection. By means of Cauchy's integral formula we obtain for (1.1)

$$
q(n)=\frac{1}{2 \pi i} \int_{c} \frac{f(x)}{x^{n+1}} d x .
$$

The path of integration may be the circle defined as $|x|=e^{-2 \pi N^{-2}}$ where $N$ is a certain positive integer at our disposal. In the usual way we divide the circle into Farey arcs $\xi_{h, k}$ of order $N$. The Farey arc $\xi_{h, k}$ is defined by

$$
x=\exp \left(2 \pi i h / k-2 \pi N^{-2}+2 \pi i \vartheta\right), \quad(h, h)=1,
$$

and

$$
-\vartheta_{1}(h, k)=\frac{h+h_{1}}{k+k_{1}}-\frac{h}{k} \leqq \vartheta \leqq \frac{h+h_{2}}{k+k_{2}}-\frac{h}{k}=\vartheta_{2}(h, k)
$$


where $h_{1} / k_{1}, h / k, h_{2} / k_{2}$ are three consecutive fractions in the Farey sequence of order $N$. It is well known that

$$
\frac{1}{k(N+k)} \leqq \vartheta_{1}(h, k)<\frac{1}{k(N+1)}, \frac{1}{k(N+k)} \leqq \vartheta_{2}(h, k)<\frac{1}{k(N+1)}
$$

We obtain then

$$
q(n)=\frac{1}{2 \pi i} \sum_{(h, k)=1,0<h \leqq k \leqq N} \int_{\xi_{h, k}} \frac{f(x)}{x^{n+1}} d x .
$$

Let $I_{1}$ and $I_{2}$ denote the sums of those terms satisfying $2 \mid k$, and $2 \nmid k$, respectively. Then, by (3.4), we have

$$
q(n)=I_{1}+I_{2}
$$

\section{Lemmas on Kloosterman's sums.}

LEMMA $4.1\left(^{5}\right)$. Let

$$
g(N, \vartheta, h, k)= \begin{cases}1 & \text { for }-\vartheta_{1}(h, k) \leqq \vartheta \leqq \vartheta_{2}(h, k), \\ 0 & \text { otherwise }\end{cases}
$$

Then

$$
g=\sum_{r=1}^{k} b_{r} e^{2 \pi i r h^{\prime} / k}
$$

where $h^{\prime}$ iz an integer satisfying

$$
h h^{\prime} \equiv 1(\bmod k)
$$

and $b_{r}$ is independent of $h$ and

$$
\sum_{r=1}^{k}\left|b_{r}\right|<\log 4 k
$$

Lemma 4.2. Let a be an absolute constant. Then

$$
\sum_{0<h \leqq a k,(h, a k)=1, h \equiv l(a)} \exp \left(\frac{2 \pi i}{a k}\left(n k+m h^{\prime}\right)\right)=O\left(k^{2 / 8+\epsilon}(n, k)^{1 / 8}\right) .
$$

LEMMA 4.3. If $k$ is even and $\omega_{h, k}$ as defined in $\$ 2$, then

$$
S_{k}=\sum_{1 \leqq h \leqq k,(k, k)=1, h h^{\prime} \equiv 1(k)} \omega_{h, k} e^{2 \pi i\left(n h+m h^{\prime}\right) / k}=O\left(k^{2 / 3+e}(n, k)^{1 / 3}\right) .
$$

Proof. For the sake of simplicity I give here only the proof of the case $24 k$.

(b) T. Estermann, Abhandlungen aus dem Mathematischen Seminar der Hamburgischen Universität, vol. 7 (1929), pp. 93, 94. 
Then

$$
S_{k}=\sum_{1 \leqq l \leqq 24,(l, 24)=1} \sum_{1 \leqq h \leqq k,(h, k)=1, h h^{\prime} \equiv 1, h \equiv l(24)} \omega_{h, k} e^{2 \pi i\left(n h+m h^{\prime}\right) / k} .
$$

The inner sum becomes a Kloosterman's sum as in Lemma 4.2. Therefore we have

$$
S_{k}=O\left(k^{2 / 3+e}(n, k)^{1 / 3}\right) .
$$

As to the proof of the other cases, nothing is difficult but a little complicated, and the foliowing fact is used: let

$$
F(h, k)=\omega_{h, k} e^{2 \pi i\left(n h+m h^{\prime}\right) / k} ;
$$

then $F(h+k, k)=F(h, k)$.

LEMMA 4.4. Let $2 \nmid k$ and $\omega_{h, k}$ be as defined in $\$ 2$, then

$$
S=\sum_{1 \leqq h \leqq k,(h, k)=1, h h^{\prime} \equiv 1(k), h^{\prime} \text { odd }} \omega_{h, k} e^{\pi i\left(2 n h+m h^{\prime}\right) / k}=O\left(k^{2 / 3+e}(h, k)^{1 / 3}\right) .
$$

The proof is similar to that of Lemma 4.3 , only notice that

$$
S=\sum_{1 \leqq h<2 k,(h, 2 k)=1, h h^{\prime} \equiv 1(2 k)}
$$

5. Lemmas from the theory of the linear transformation of the elliptic modular functions.

LEMma 5.1. Suppose that $2 \nmid h, 2 \mid k$; that $h^{\prime}$ is a positive integer satisfying $h h^{\prime} \equiv 1(\bmod k)$; that $\omega_{h, k}$ is defined in $\$ 2 ;$ and that

$$
x=\exp \left(-\frac{2 \pi z}{k}+\frac{2 h \pi i}{k}\right), \quad x^{\prime}=\exp \left(-\frac{2 \pi}{k z}-\frac{2 h^{\prime} \pi i}{k}\right),
$$

where the real part of $z$ is positive. Then

$$
f(x)=\omega_{h, k} \exp \left(-\frac{\pi}{12 k z}+\frac{\pi z}{12 k}\right) f\left(x^{\prime}\right) .
$$

Proof. If we take $a=h, b=-k, c=\left(1-h h^{\prime}\right) / k, d=h^{\prime}$, so that $a d-b c=1$, and write

$$
\begin{array}{ll}
x=q^{2}=e^{2 \pi i r}, & x^{\prime}=Q^{2}=e^{2 \pi i T,} \\
\tau=(h+i z) / k, & T=\left(-h^{\prime}+i / z\right) / k,
\end{array}
$$

then we can easily verify that

$$
T=\frac{c+d \tau}{a+b \tau}
$$


Also, in the notation of Tannery and Molk, we obtain

$$
f(x)=\frac{1}{2^{1 / 3}} q^{-1 / 12} \frac{\phi(\tau)}{\chi(\tau)}, \quad f\left(x^{\prime}\right)=\frac{1}{2^{1 / 3}} Q^{-1 / 12} \frac{\phi(T)}{\chi(T)} .
$$

Then

$$
\begin{aligned}
f\left(x^{\prime}\right)= & \frac{1}{2^{1 / 3}} Q^{-1 / 12} \frac{\phi(T)}{\chi(T)}=\exp \left(\pi i \left(\frac{1}{8}\left(d^{2}-1\right)(\dot{c}-1)+\frac{c d}{8}\right.\right. \\
& \left.\left.\quad-\frac{(b-c)(b c d-a)}{24}\right)\right) \frac{1}{2^{i / 3}} Q^{-1 / 12} \frac{\phi(\tau)}{\chi(\tau)} \\
= & \exp \left(\pi i\left(\frac{1}{8}\left(d^{2}-1\right)(c-1)+\frac{c d}{8}-\frac{(b-c)(b c d-a)}{24}\right)\right) q^{1 / 12} Q^{-1 / 12} f(x) \\
= & \exp \left(\pi i\left(\frac{1}{8}\left(d^{2}-1\right)(c-1)+\frac{c d}{8}-\frac{(b-c)(b c d-a)}{24}\right)\right) \\
& \cdot \exp \left(\frac{\pi}{12 k}\left(\frac{1}{z}-z\right)\right) \exp \left(\frac{\pi i}{12 k}\left(h+h^{\prime}\right)\right) f(x) .
\end{aligned}
$$

LEMMA 5.2. Suppose that $2 \nmid h k$ and $h h^{\prime} \equiv 1(\bmod 2 k)$, that

Then

$$
f_{1}(x)=\prod_{1}^{\infty}\left(1+x^{n-1 / 2}\right)=1+\sum_{n=1}^{\infty} q_{1}(n) x^{n / 2} .
$$

$$
f(x)=\frac{\omega_{h, k}}{2^{1 / 2}} \exp \left(\frac{\pi}{12 k}\left(z+\frac{1}{2 z}\right)\right) f_{\mathrm{i}}\left(x^{\prime}\right) .
$$

Proof. As in Lemma 5.1, we have

$$
\begin{aligned}
f_{1}(x)= & f_{1}\left(q^{2}\right)=\Pi\left(1+q^{2 n-1}\right)=2^{1 / 6} q^{1 / 24} \frac{1}{\chi(\tau)} \\
f_{1}\left(x^{\prime}\right)= & 2^{1 / 8} Q^{1 / 24} \frac{1}{\chi(T)}=2^{1 / 6} Q^{1 / 24} \exp \left(-\frac{(b-c)(a b c-d)}{24} \pi i\right) \frac{\phi(\tau)}{\chi(\tau)} \\
= & 2^{1 / 6} Q^{1 / 24} \exp \left(-\frac{(b-c)(a b c-d)}{24} \pi i\right) 2^{1 / 3} q^{1 / 12} f(x) \\
= & \exp \left(-\frac{(b-c)(a b c-d)}{24} \pi i\right) 2^{1 / 2} \\
& \cdot \exp \left(\frac{\pi i}{24}\left(-\frac{h^{\prime}}{k}+\frac{i}{k z}+\frac{2 h}{k}+\frac{2 i z}{k}\right)\right) f(x) .
\end{aligned}
$$


Lemma 5.3. Suppose that $2\left|h, 2 \nmid k, h h^{\prime} \equiv 1(\bmod k), 2\right| h^{\prime}$ and suppose that

$$
f_{2}(x)=\prod_{1}^{\infty}\left(1-x^{n-1 / 2}\right)=1+\sum q_{2}(n) x^{n / 2} .
$$

Then

$$
f(x)=\frac{\omega_{h, k}}{2^{1 / 2}} \exp \left(\frac{\pi}{12 k}\left(z+\frac{1}{2 z}\right)\right) f_{2}\left(x^{\prime}\right) .
$$

Proof. We take

$$
a=-h, \quad b=k, \quad c=\left(h h^{\prime}-1\right) / k, \quad d=-h^{\prime} .
$$

Then

$$
\begin{aligned}
f_{2}\left(x^{\prime}\right) & =f_{2}\left(Q^{2}\right)=2^{1 / 8} Q^{1 / 24} \frac{\psi(T)}{\chi(T)} \\
& =2^{1 / 8} Q^{1 / 24} \exp \left(\frac{\pi i}{2}\left(\frac{b^{2}-1}{4}+\frac{a b}{4}-\frac{(a+d)(a b d-c)}{12}\right)\right) \frac{\phi(\tau)}{\chi(\tau)} \\
& =2^{1 / 2} \exp \left(\frac{\pi i}{2}\left(\frac{b^{2}-1}{4}+\frac{a b}{4}-\frac{(a+d)(a b d-c)}{12}\right)\right) Q^{1 / 24} q^{1 / 12} f(x) .
\end{aligned}
$$

6. Approximation of the integrand. Let

Then

$$
z=k\left(N^{-2}-i \vartheta\right) .
$$

$$
\begin{gathered}
I_{1}=\sum_{1 \leqq k \leqq N, 2 \mid k} \sum_{(h, k)=1,0<h<k} \int_{-k^{-1}(N+1)-1}^{k^{-1}(N+1)^{-1}} g(\vartheta) f\left(e^{(2 \pi i h-2 \pi z) / k}\right) e^{-2 \pi i h n / k+2 \pi z n / k} d \vartheta \\
=\sum_{1 \leqq k \leqq N, 2 \mid k} \sum_{(h, k)=1,0<h<k} \int_{-k^{-1}(N+1)-1}^{k^{-1}(N+1)^{-1}} g(\vartheta) \omega_{i h, k} e^{(\pi / 12 k)(z-1 / z)} \\
=\sum_{1 \leqq k \leqq N, 2 \mid k} \sum_{(h, k)=1,0<h<k} \int_{\left.-k^{\prime}\right) e^{-2 \pi i h n / k+2 \pi z \pi / k} d \vartheta}^{k^{-1}(N+1)^{-1}} g(\vartheta+1)-1 \\
\cdot e^{(\pi / 12 k)(z-1 / z)-2 \pi i h n / k+2 \pi z n / k} \sum_{\nu=0}^{\infty} q(\nu) e^{-\left(2 \pi / k z+2 h^{\prime} \pi i / k\right) \nu} d \vartheta \\
\sum_{1 \leqq k \leqq N, 2 \mid k} \sum_{(h, k)=1,0<h<k} \int_{-k^{-1}(N+1)-1}^{k^{-1}(N+1)^{-1}} \sum_{\nu=0}^{\infty} q(\nu) e^{-(2 \pi / k z)(i+1 / 24)+(2 \pi z / k)(n+1 / 24)} \\
\cdot \sum_{r=1}^{k} b_{r} e^{2 \pi i+h^{\prime} / k} \omega_{h, k} e^{-2 \pi i h n / k-2 \pi i h^{\prime} \nu / k} d \vartheta .
\end{gathered}
$$


Since $(1 / k) R(1 / z) \geqq \frac{1}{2}$, we have

$$
\begin{aligned}
\left|I_{1}\right| \leqq & \sum_{1 \leqq k \leqq N, 2 \mid k} \int_{-k^{-1}(N+1)-1}^{k^{-1}(N+1)^{-1}} \sum_{\nu=0}^{\infty} q(\nu) \\
& \cdot \exp \left\{-\frac{2 \pi}{k}\left(\nu+\frac{1}{24}\right) R \frac{1}{z}+\frac{2 \pi}{k}\left(n+\frac{1}{24}\right) R z\right\} \\
& \sum_{r=1}^{k}\left|b_{r}\right|\left|\sum_{(h, k)=1} \omega_{h, k} e^{-2 \pi i h n / k+2 h^{\prime}(r-v) \pi i / k}\right| d \vartheta \\
= & O\left(\sum_{k=1}^{N} \int_{-k-1(N+1)-1}^{k^{-1}(N+1)^{-1}} \sum_{\nu=0}^{\infty} q(\nu) e^{-\pi(\nu+1 / 24)} \sum_{r=1}^{k}\left|b_{r}\right| k^{2 / 3} d \vartheta\right) \\
= & O\left(\sum_{k=1}^{N} \log k \cdot k^{2 / 3} \frac{1}{k N}\right)=O\left(\frac{1}{N} \sum_{k=1}^{N} k^{-1 / 3+\bullet}\right) \\
= & O\left(N^{-1 / 3+\iota) .}\right.
\end{aligned}
$$

Let

$$
\begin{aligned}
J=\frac{1}{2^{1 / 2}} \sum_{k=1, k \text { odd }}^{N} \sum_{(h, k)=1,0<h \leqq k} \int_{-k-1(N+1)-1} \int^{k^{-1}(N+1)^{-1}} g(\vartheta) \omega_{h, k} \\
\cdot e^{(\pi / 24 k)(2 z+1 / z)-2 \pi i h n / k+2 \pi z n / k} d \vartheta .
\end{aligned}
$$

The same method will give us that $\left|I_{2}-J\right|=O\left(N^{-1 / 8+c}\right)$.

7. A contour integration. Let $w=N^{-2}-i \vartheta$. Then

$$
\begin{aligned}
J= & \frac{-i}{2^{1 / 2}} \sum_{1 \leqq k \leqq N, k \text { odd }} \sum_{(h, k)=1,0<h \leqq k} \omega_{h, k} e^{-2 \pi i h n / k} \int_{N-2-w_{2}}^{N^{-2}+w_{1}} e^{2 \pi w(n+1 / 24)+\pi / 24 k^{2} w} d w \\
= & \frac{i}{2^{1 / 2}} \sum_{1 \leqq k \leqq N, k \text { odd }} \sum_{(h, k)=1,0<h \leqq k} \omega_{h, k} e^{-2 \pi i h n / k}\left(\int_{N-2+w_{1}}^{N^{-2}+i k^{-1}(N+1)^{-1}}\right. \\
& +\int_{N-2+i k-1(N+1)-1}^{-N^{-2}+i k^{-1}(N+1)^{-1}}+\int_{-N-2+i k-1(N+1)-1}^{-N^{-2}-i k^{-1}(N+1)^{-1}}+\int_{-N-2-i k-1(N+1)-1}^{N^{-2}+i k^{-1}(N+1)^{-}} \\
& \left.+\int_{N^{-2-i k-1}(N+1)}^{N^{-2}-w_{2}}-2 \pi i \text { Residue at } 0\right) \\
= & K_{1}+K_{2}+K_{3}+K_{4}+K_{6}+L \text { (say). }
\end{aligned}
$$

We have

$$
\begin{aligned}
K_{1}= & \frac{i}{2^{1 / 2}} \sum_{1 \leqq k \leqq N, k \text { odd }} \sum_{(h, k)=1,0<h \leqq k} \omega_{h, k} e^{-2 \pi i h n / k} \\
& \cdot \int_{N-2+i k-1(N+k)-1}^{N^{-2}+i k^{-1}(N+1)^{-1}} g(\vartheta) e^{2 \pi w(n+1 / 24)+\pi / 24 k^{2} w} d w .
\end{aligned}
$$


By Lemma 3.1, we have

$$
\begin{aligned}
K_{1} & =O\left(\sum_{1 \leqq k \leqq N, k \text { odd }} k^{2 / 3+\bullet} \int_{k^{-1}(N+k)-1}^{k^{-1}(N+1)^{-}} \exp \left\{2 \pi\left(n+\frac{1}{24}\right) R w+\frac{\pi}{24 k^{2}} R \frac{1}{w}\right\} d w\right. \\
& =O\left(\sum_{k=1}^{N} k^{2 / 3+๕} e^{-2 \pi n N^{-2}} \int_{k-1(N+k)-1}^{k^{-1}(N+1)^{-1}} d \vartheta\right) \\
& =O\left(N^{-1 / 3+\epsilon)} .\right.
\end{aligned}
$$

Similar result holds for $K_{5}$.

We have

$$
R \frac{1}{k^{2} w}=\frac{N^{-2}}{k^{2} N^{-2}+N^{2}}, \quad K_{2}=O\left(\sum_{k=1}^{N} N^{-2} k^{2 / 3+c}\right)=O\left(N^{-1 / 3+\bullet}\right) .
$$

Similar result holds for $K_{4}$.

Applying again Kloosterman's argument to $K_{3}$, we have also $K_{3}=O\left(N^{-1 / 3}\right)$.

Finally we find the residue of $\exp \left(2 \pi w(n+1 / 24)+\pi / 24 k^{2} w\right)$ at $w=0$. We have the expansion

The residue is, therefore,

$$
\begin{aligned}
e^{2 \pi w(n+1 / 24)} & =\sum_{\nu=1}^{\infty} \frac{(2 \pi w(n+1 / 24))^{\nu}}{\nu !} \\
e^{\pi / 24 k^{2} w} & =\sum_{\mu=1}^{\infty} \frac{1}{\mu !}\left(\frac{\pi}{24 k^{2} w}\right)^{\mu} .
\end{aligned}
$$

$$
\begin{aligned}
\sum_{\mu=1}^{\infty} \frac{1}{\mu !}\left(\frac{\pi}{24 k^{2}}\right)^{\mu} \frac{1}{(\mu-1) !} & \left(2 \pi\left(n+\frac{1}{2 \pi}\right)\right)^{\mu-1} \\
= & \frac{1}{2 \pi} \frac{d}{d n} \sum_{\mu=1}^{\infty} \frac{1}{(\mu !)^{2}}\left(\frac{\pi}{24 k^{2}}\right)^{\mu}\left(2 \pi\left(n+\frac{1}{2 \pi}\right)\right)^{\mu} \\
& =\frac{1}{2 \pi} \frac{d}{d n} \sum_{\mu=1}^{\infty} \frac{1}{2^{2 \mu}(\mu !)^{2}}\left(\frac{\pi}{k}\left\{\frac{1}{3}\left(n+\frac{1}{2 \pi}\right)\right\}^{1 / 2}\right)^{2 \mu} \\
& =\frac{1}{2 \pi} \frac{d}{d n} J_{0}\left(\frac{i \pi}{k}\left\{\frac{1}{3}\left(n+\frac{1}{2 \pi}\right)\right\}^{1 / 2}\right) .
\end{aligned}
$$

Therefore

$$
\begin{aligned}
q(n)= & \frac{1}{2^{1 / 2}} \sum_{k=1, k \text { odd }}^{N} \sum_{(h, k)=1,0<h \leqq k} \omega_{h, k} e^{-2 \pi i h n / k} \frac{d}{d n} J_{0}\left(\frac{i \pi}{k}\left\{\frac{1}{3}\left(n+\frac{1}{2 \pi}\right)\right\}^{1 / 2}\right) \\
& +O\left(N^{-1 / 8+e)} .\right.
\end{aligned}
$$

Let $N \rightarrow \infty$; we obtain the theorem.

National Tsing Hua University,

Kunming, Yünnan, China 